\title{
Dual-pump CARS of Air in a Heated Pressure Vessel up to 55 Bar and $1300 \mathrm{~K}$
}

\author{
Luca Cantu*, Emanuela Gallo*, Andrew D. Cutler ${ }^{\dagger}$, \\ The George Washington University, Mechanical and Aerospace Engineering, \\ 1 Old Oyster Point Road, Suite 200, Newport News, VA, 23602 \\ Paul M. Danehy \\ NASA Langley Research Center, Hampton, VA, 23681
}

\begin{abstract}
Dual-pump Coherent anti-Stokes Raman scattering (CARS) measurements have been performed in a heated pressure vessel at NASA Langley Research Center. Each measurement, consisting of $\mathbf{5 0 0}$ single shot spectra, was recorded at a fixed location in dry air at various pressures and temperatures, in a range of $0.03-55 \times 10^{5} \mathrm{~Pa}$ and $300-1373 \mathrm{~K}$, where the temperature was varied using an electric heater. The maximum output power of the electric heater limited the combinations of pressures and temperatures that could be obtained. Charts of CARS signal versus temperature (at constant pressure) and signal versus pressure (at constant temperature) are presented and fit with an empirical model to validate the range of capability of the dual-pump CARS technique; averaged spectra at different conditions of pressure and temperature are also shown.
\end{abstract}

\section{Introduction}

Coherent anti-Stokes Raman scattering (CARS $)^{1,2}$ is a non-linear spectroscopic technique that provides spatially and temporally resolved temperature and species concentration by probing molecular Raman shifts. Three coherent laser beams (pump, Stokes and probe) are focused and crossed in the region of interest generating a CARS signal beam. The frequencies of the three beams are chosen such that their interaction excites the molecular rotationalvibrational transitions of the species of interest. In dual-pump CARS, ${ }^{2,3}$ pump and probe beams exchange roles during the measurement to detect simultaneously two different Raman shifts (in this case, in the vicinity of $\mathrm{N}_{2}$ and $\mathrm{O}_{2}$ resonances), as shown in Figure 1:
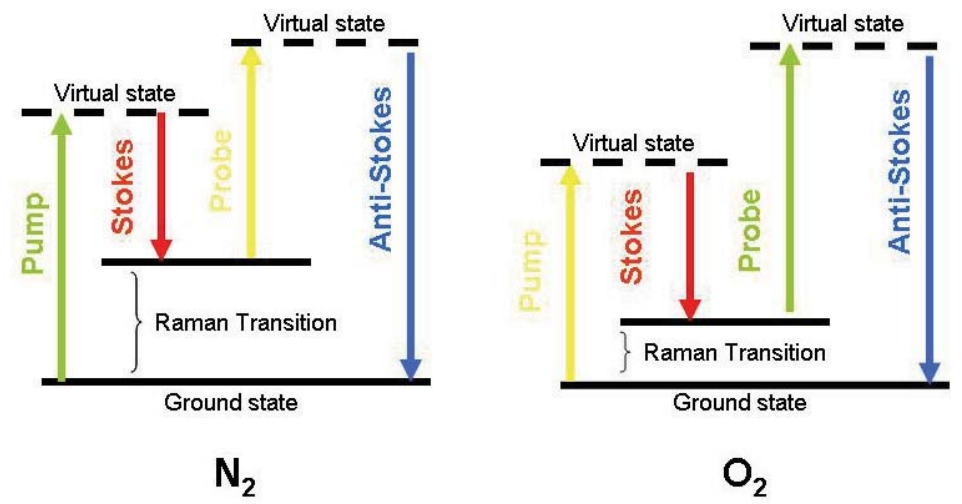

Figure 1. Dual-pump CARS energy level diagrams for $\mathrm{N}_{2}$ and $\mathrm{O}_{2}$

To generate a high-intensity and coherent blue signal beam, constructive laser beam interference is required at the measurement point, where the three laser beams are crossed. In this experiment, a planar BOXCAR configuration

* Ph.D. Candidate, Member AIAA

${ }^{\dagger}$ Professor. Associate Fellow AIAA

${ }^{\ddagger}$ Research Scientist. Associate Fellow AIAA 
was used to achieve phase matching. The resulting blue signal beam, generated in the forward direction (on top of the pump beam), carries the Raman spectra of the probed species. Processing of the spectra allows temperature to be obtained from the spectral shape, while species concentrations affect signal intensity, since the non-linear third-order susceptibility is dependent on both density and temperature ${ }^{2}$. Due to its highly coherent signal and the capability to measure simultaneously temperature and species concentrations with small optical access, CARS is a useful measurement tool in harsh environments. Of particular interest is the manufacturing of boron nitride nanotubes (BNNT), ${ }^{4}$ which occurs at high pressure $\left(2 \times 10^{5}\right.$ to $20 \times 10^{5} \mathrm{~Pa}$ ) and high temperature (up to $4600 \mathrm{~K}$ ) in an $\mathrm{N}_{2}$ atmosphere. ${ }^{5}$

In this paper, combined effects of high pressure and high temperature on CARS are presented. A study was conducted in dry air: the pressure was varied from $0.5 \times 10^{5} \mathrm{~Pa}$ to $55 \times 10^{5} \mathrm{~Pa}$, and the temperature varied from room temperature $(300 \mathrm{~K})$ to nearly $1400 \mathrm{~K}$. At high pressure, previous work predicted that the CARS signal would increase with the square of the pressure. ${ }^{6}$ Furthermore, according to Dreier et al., ${ }^{7}$, who studied collisional narrowing of $\mathrm{O}_{2}$ and $\mathrm{N}_{2}$ at room temperature and pressure up to $2500 \times 10^{5} \mathrm{~Pa}$, spectral lines significantly collapse together due to collisional narrowing. At high temperature, additional vibrational modes become excited, and signal is expected to drop with ${ }^{6} T^{3.5}$. Seeger et al. ${ }^{8}$ investigated both high temperature (up to $700 \mathrm{~K}$ ) and high pressure (up to $150 \times 10^{5}$ $\mathrm{Pa}$ ); they used pure rotational CARS, a technique that provides accurate results at high pressure since spectra are almost free from collisional narrowing. However, pure rotational CARS is less sensitive to temperature than dualpump CARS at high temperatures, and it is not able to provide information about vibrational transitions.

Dual-pump CARS experiments have been conducted at NASA Langley Research Center in a heated pressure vessel owned by the National Institute of Aerospace (NIA) in Hampton VA, to demonstrate the capability of this technique to obtain reliable signal in high temperature and high pressure environments. This vessel will be used in the manufacture of BNNT in the presence of $\mathrm{N}_{2} \cdot{ }^{4}$

\section{Experimental Method}

The lasers and optical systems used to generate the three CARS laser beams are mounted on a mobile cart described in detail by Cutler et al. ${ }^{9,10}$ As shown in Fig. 2, the three laser beams exit the cart- the pump beam (green), the Stokes beam (red), and the probe beam (yellow, under the red beam) - and are sent to two optical boards.

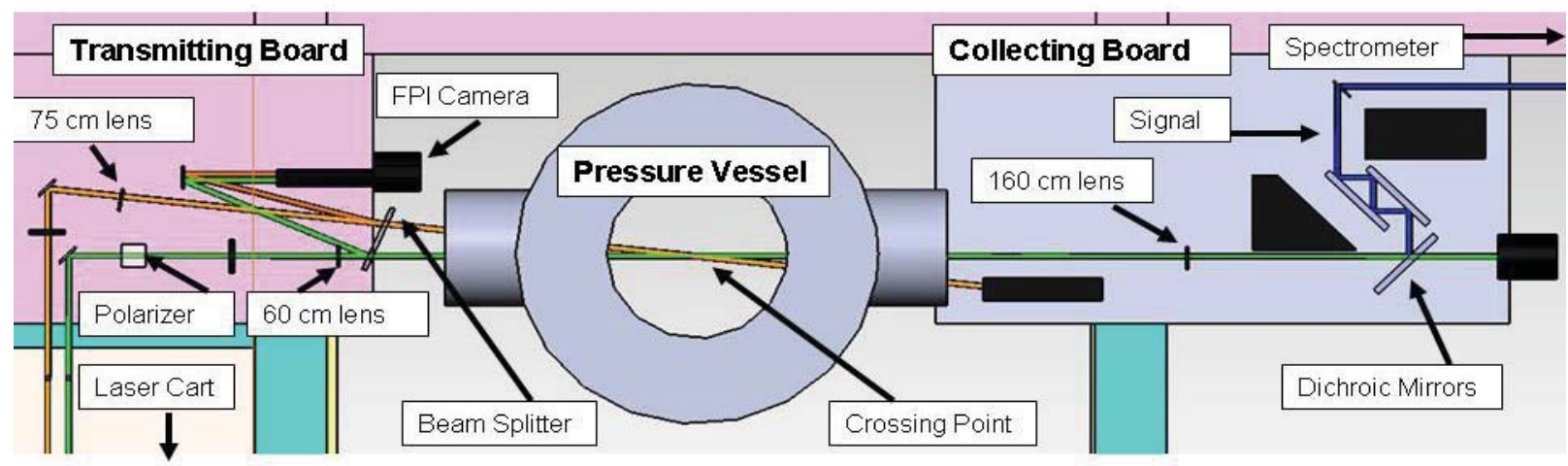

Figure 2. Experimental Layout. Transmitting and Collecting Boards optical setup

The transmitting board, placed before the pressure vessel, is designed to receive all laser beams and send them focused to the measurement volume inside the vessel. The green beam is passing through a polarizer, which guarantees the correct polarization of the beam, and a tilted lens $(60 \mathrm{~cm}$ focal length) that focuses and shapes the beam into the measurement volume. This lens provides an elliptic shape to the green beam to reduce the sensitivity to misalignment of the crossing point. ${ }^{11}$ The red and yellow beams pass together through a $75 \mathrm{~cm}$ focal length lens, which focuses them at the crossing point. Since the measurement volume cannot be directly observed for alignment purposes, a separate external beam crossing, which is an image of the crossing in the vessel, is monitored. A removable beam splitter is placed before the entrance of the pressure vessel and deflects a small percentage of the beams to the focal plane imaging (FPI camera); the camera is focused to image the plane where the beams cross and 
are focused. Figure 3 shows four images from the FPI camera: the first three images show one beam at time, and the last image shows all three beams together, demonstrating that the beams are overlapped at the desired crossing point. This method was applied to align all three beams and cross them in the measurement volume without removing the pressure vessel from its position.
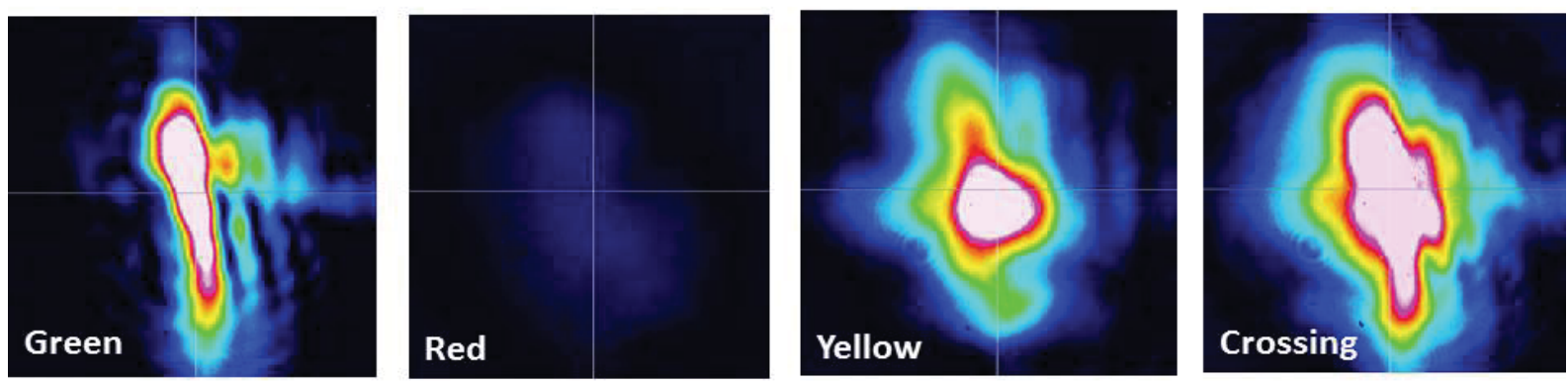

Figure 3. Focal Plane Imaging alignment images. Each beam is aligned individually at the center of the FPI camera, resulting in crossing all of them in the same place.

The collection optics board, placed after the pressure vessel, was designed to receive the signal (blue) created at the crossing point, separate it from the overlapped green beam, and send it to the spectrometer. Red and yellow beams were dumped into a beam block. Overlapped green and signal beams were first collimated by a $160 \mathrm{~cm}$ focal length lens, then sent through a series of dichroic mirrors that separate most of the green beam (sent into a beam dump) from the blue one. The CARS signal then passed through a series of mirrors, additional filters to eliminate residual green light, a polarizer, and was then focused by a $5 \mathrm{~cm}$ lens to the entrance of a 1 meter spectrometer. All the spectra were acquired on a CCD camera. To prevent camera saturation (above 65,535 counts a.u.), additional filters were placed in front of the last lens as needed.

The pressure vessel was placed between the two optical boards and provided optical access for the laser beams on both sides. A cylindrical $23 \mathrm{~cm}$ long fiberglass electrical resistance heater (maximum power $220 \mathrm{~W}$ ) with a 1.9 $\mathrm{cm}$ inside diameter was placed inside the vessel. A hole was cut at each end of the heater, roughly $1.3 \mathrm{~cm}$ wide and 1 $\mathrm{mm}$ high, to allow the CARS laser beams to pass through. A thermocouple was inserted into the side wall of the heater at the center, penetrating about $1 \mathrm{~mm}$ into the inside diameter. A temperature controller used this thermocouple in a feedback loop to maintain a constant temperature in the heater.

CARS measurements were performed at a fixed location inside the heated pressure vessel. The temperature was limited to $1400 \mathrm{~K}$ to prevent damage to the heater. The pressure inside the vessel was limited to $55 \times 10^{5} \mathrm{~Pa}$ by its design and by a pressure-relief safety valve. The heater was not powerful enough to achieve its maximum temperature over the full pressure range. Spectra were collected when the CARS signal-to-noise ratio was high enough (above 5000 counts a.u. of the $\mathrm{N}_{2}$ peak on the camera) to perform a reliable data analysis. A few points were randomly repeated for statistical purposes.

All the preliminary alignment was done using continuous wave $(\mathrm{CW})$ pointing lasers (energy less than $5 \mathrm{~mJ}$ ) collinear with the high power lasers. A series of apertures placed in the optical paths provided target references for both low power and high power laser alignments. The green pointing laser was also used to align the signal to the spectrometer by removing the last two filters.

The CARS signal beam was observed to go out of alignment with the collection optics when the cell was operating at both high temperature and high pressure. The CARS laser beams and the alignment lasers were both also observed to deflect downward significantly under these conditions. This downward deflection was postulated to be due to stratification of the gas within the heater (hotter towards the top) resulted in vertical refractive index gradients. This was a very large effect, with deflection at the collimating lens on the collecting board as much as 2.5$5 \mathrm{~cm}$. To overcome this issue, the CARS signal was re-aligned for every new combination of temperature and pressure. This alignment depended on the signal being collinear to the green beam. The initial alignment of the signal to the spectrometer (prior to the operation of the heater) was with a green $\mathrm{CW}$ laser pointer, which was previously set to follow the green beam. Irises were then set along the path between the collimating lens on the collection optics board and the spectrometer. When the beams were deflected by operation of the heater, the height 
of the collimating lens was adjusted by centering it to the laser pointer, and then the subsequent mirror on the beam path was adjusted to bring the laser pointer beam back to the irises. Fine adjustments were made by observing the signal beam on the spectrometer detector. In some cases, a small adjustable periscope was placed in front of the collimating lens to correct for large beam displacements. The focal plane imaging system (Figure 3) was used repeatedly to make sure that the input beams stayed aligned to the original path throughout the experiment.

Tests were performed to confirm uniform temperature inside the heater in a region close to the measurement volume. This was done only at atmospheric pressure, and with one window of the pressure vessel removed to allow access for the measurement. A type $\mathrm{K}$ thermocouple probe was inserted from the open end into the heater and translated along the heater axis to measure the longitudinal profile of temperature. The results were compared with the nominal temperature measured through the sidewall of the heater, $11.4 \mathrm{~cm}$ from the heater end. As shown in Figure 4, a uniform temperature region was found from 8.9 to $13.9 \mathrm{~cm}$ (laser crossing point was set at $11.4 \pm 0.5 \mathrm{~cm}$ ) for several different nominal temperatures. Temperature variation is less than $20 \mathrm{~K}$ per cm, and in agreement with the nominal temperature measured by the temperature controller.

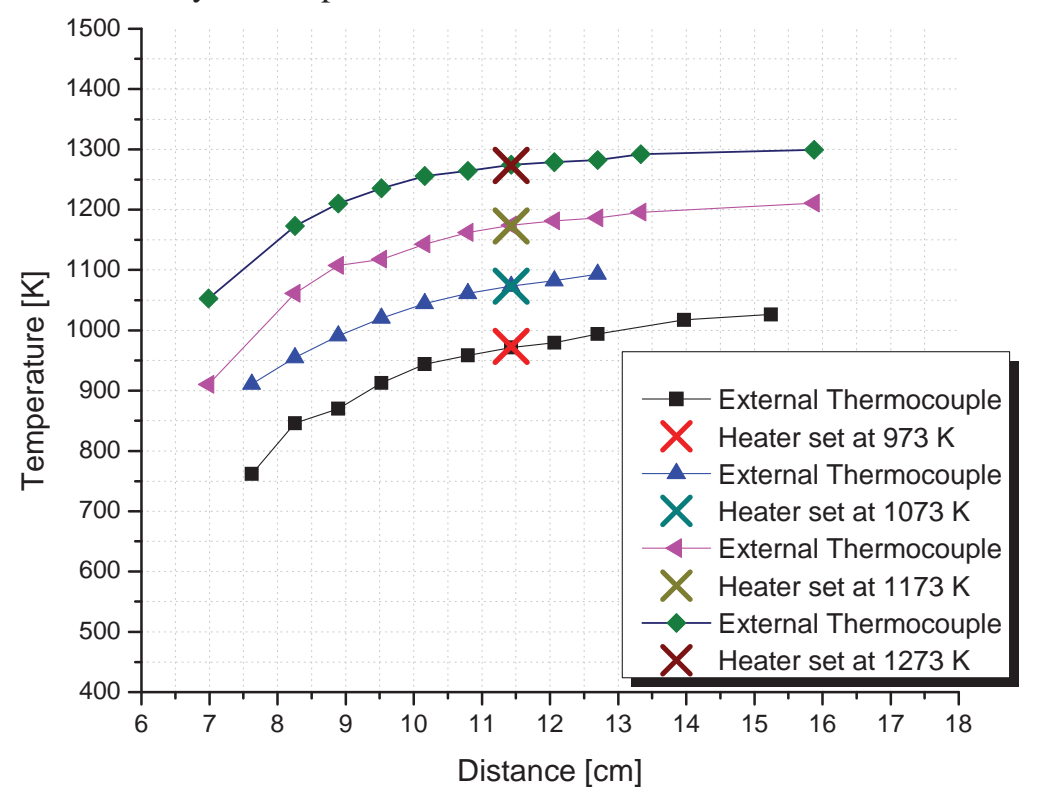

Figure 4. Temperature uniformity inside heater. Uniform region inside the heater tested with an external type K thermocouple. Measurements performed at atmospheric pressure.

\section{Data Analysis}

A statistically high number (500 single shots) of spectra were collected at several combinations of pressure and temperature. All the measured spectra were preprocessed to obtain the CARS susceptibility spectra and then compared to theoretical spectra. The preprocessing consisted of subtracting the background from each single shot taken and removing any possible shots affected by laser-induced breakdown. (Laser-induced breakdown was determined to be present if the baseline of the spectrum was more than a threshold.) Spectra were then averaged and normalized by a reference non-resonant (Argon) spectrum in order to remove the effects of the Stokes laser spectrum, as described in detail by Cutler et al. ${ }^{12,13}$

Collected dual-pump spectra contained both $\mathrm{N}_{2}$ and $\mathrm{O}_{2}$ Q-branch resonances. However, only the $\mathrm{N}_{2}$ part of the spectrum has been considered in our analysis. The $\mathrm{O}_{2}$ part of the spectrum was omitted from analysis because it is not relevant to the BNNT-manufacturing application. Also, the Modified Exponential Gap (MEG) model discussed below has not been implemented for $\mathrm{O}_{2}$ in the CARSFT code used to analyze these spectra.

Knowing the environmental conditions and chemical composition for each measurement, theoretical spectra were created for comparison with data using Sandia CARSFT code $^{14}$. In previous experiments ${ }^{9-13}$, the Voigt model was proven to be a reliable model to fit CARS spectra in combustion environments; however, the Voigt model was found to be less accurate in high pressure environments, so the MEG model was chosen to better capture the 
collisional narrowing phenomena occurring at high pressure conditions, as described by Woyde and Stricker. ${ }^{15}$ Unfortunately, the MEG model failed (in our version of CARSFT) for dual-pump CARS. [We do not know if this is an error due to changes we may have made to CARSFT or was originally present in the dual-pump version of CARSFT that we obtained from Lucht ${ }^{3}$. Theoretical dual-pump spectra were therefore calculated with MEG by running a single-pump calculation with modified input data.

The basis for this calculation is as follows. The complex CARS signal amplitude, $X$, for single pump-CARS in air (modeled as $79 \% \mathrm{~N}_{2}$ and $21 \% \mathrm{O}_{2}$ ) is represented by Equation 1 (where $X$ is proportional to CARS susceptibility):

$$
X=0.79 X_{N 2}+0.21 X_{O 2}
$$

Dual pump CARS (Equation 2) contains the sum of contributions, one from each of the CARS processes illustrated in Figure 1, (where the subscripts 1 and 2 indicated the two CARS processes denoted by the left and right sides of Figure 1):

$$
X=\left(\frac{0.79 X_{N 2}+0.21 X_{O 2}}{2}\right)_{1}+\left(\frac{0.79 X_{N 2}+0.21 X_{O 2}}{2}\right)_{2}
$$

If a frequency range is considered in which only $\mathrm{N}_{2}$ in the first process is resonant, and $\mathrm{O}_{2}$ for the first process and both $\mathrm{N}_{2}$ and $\mathrm{O}_{2}$ for the second process are all non-resonant, then Equation 2 may be rewritten to look like a single pump CARS equation:

$$
\begin{gathered}
X=\left(\frac{0.79 X_{\mathrm{N} 2}+0.21 X_{\mathrm{O} 2, \mathrm{NR}}}{2}\right)_{1}+\left(\frac{0.79 X_{\mathrm{N} 2, \mathrm{NR}}+0.21 X_{\mathrm{O} 2, \mathrm{NR}}}{2}\right)_{2}=0.395 X_{\mathrm{N} 2}+0.605 X_{\text {buffer }} \\
\text { where } X_{\text {buffer }}=\left(\frac{0.395 X_{\mathrm{N} 2, \mathrm{NR}}+0.21 X_{O 2, \mathrm{NR}}}{0.605}\right)
\end{gathered}
$$

The single pump CARS calculation for air was thus performed by setting the mole fraction of resonant $\mathrm{N}_{2}$ to 0.395 , treating the remaining 0.605 mole fraction of non-resonant gases as a mixture of buffer gases, and calculating the non-resonant susceptibility of these "buffer" gases using Equation 3 (by substituting the corresponding non-resonant susceptibilities for the $X$ 's). The validity of this approach was tested by performing a dual pump calculation using the Voigt model with the correct non-resonant gas susceptibilities and mole fractions and comparing it to a single pump calculation with the new inputs. The results were identical, thus verifying the method.

\section{Results}

Bultitude and co-workers ${ }^{6}$ used CARSFT to compute $\mathrm{N}_{2}$ CARS spectra for a range of temperatures and pressures applicable to high-enthalpy shock tunnel flow. The peak intensity in the CARS spectrum was graphed versus temperature for a variety of pressures (shown in Figure 5). The signal intensity is observed to increase with pressure and decrease with temperature. The following approximate scalings for the CARS signal intensity were noted in the paper, although the delineation between low and high pressure was not specified:

$$
\begin{array}{ll}
\text { CARS Signal } \sim P^{1} T^{3.5} & \text { (low pressure) } \\
\text { CARS Signal } \sim P^{2} T^{3.5} & \text { (high pressure) }
\end{array}
$$

Bultitude used the Voigt line shape model in his computations. However, as previously discussed, the MEG line shape model is known to provide more accurate calculations of the spectra; these calculations were repeated using the MEG model and least squares fitted to the same power form to obtain:

$$
\text { CARS Signal } \left.\sim P^{1.9} T^{3.7} \quad \text { (pressure greater than } 1 \mathrm{~atm}\right)
$$

The standard error in the fit was $\sim 13 \%$ of the measured values at each fitted point. The uncertainty in the pressure and temperature exponents is $6 \%$ and $3 \%$ respectively. This uncertainty was obtained by removing groups of calculated points (for example, all data at a certain temperature) and re-fitting the data. 
These scaling rules assume that the CARSFT program accurately predicts the intensity of the CARS signal. A major goal of the present work was to investigate whether these scalings were valid, so that they could be used to predict the CARS signal intensity in proposed BNNT manufacturing experiments.

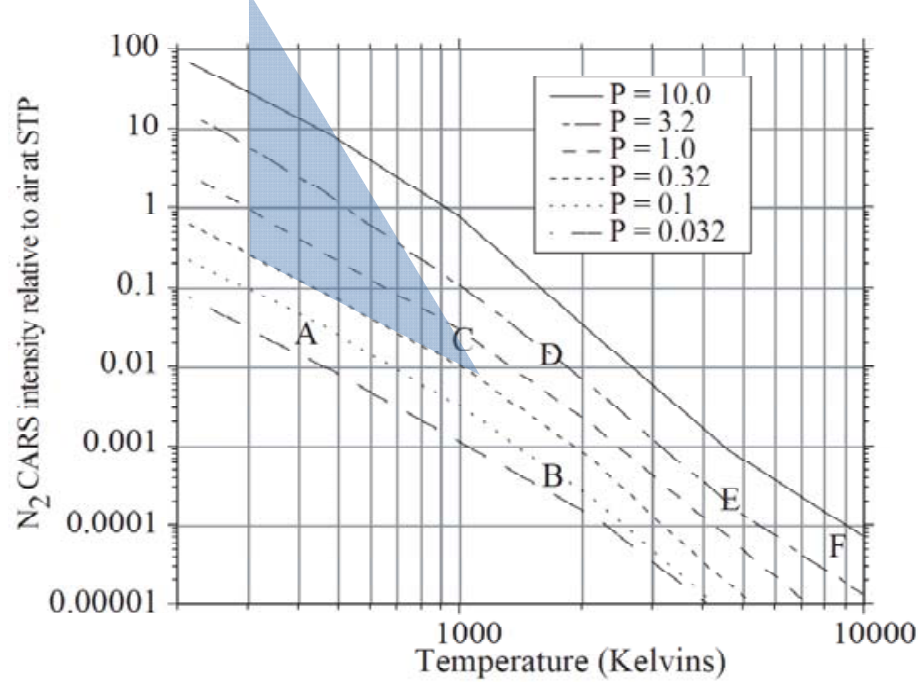

Figure 5. Predicted CARS signal intensity versus temperature. $\mathrm{N}_{2}$ CARS signal intensities previously predicted from CARSFT by Bultitude and coworkers6 (reprinted and modified with permission). Letters A-F indicated test conditions that could be achieved in the ANU T3 shock tunnel. Pressure is in atmospheres. Shaded region indicates approximate range of measurements in current work.

In the current experiment, calibrated neutral density filters were used to prevent saturation of the detector. Using this approach, measured CARS intensities could be put on an absolute intensity scale, normized to 1 at room temperature and atmospheric pressure (as in Figure 5). Figure 6 shows the signal intensity versus pressure, graphed for various temperatures. For pressures $<14 \times 10^{5} \mathrm{~Pa}$, as the pressure increases, the CARS signal increases linearly on $\log \log$ axes, indicating power law behavior. However, for each temperature, above $14 \times 10^{5} \mathrm{~Pa}$, the signal intensity stays roughly constant, at approximately the value obtained at $14 \times 10^{5} \mathrm{~Pa}$. This unexpected behavior is not predicted by the initial CARSFT computations by Bultitude and co-workers, who did not use a collisional narrowing model.6 Nor is it predicted by our computations using the MEG model. However, Kulatilaka et al. ${ }^{16}$ did observe this leveling off of CARS signal at high pressure.

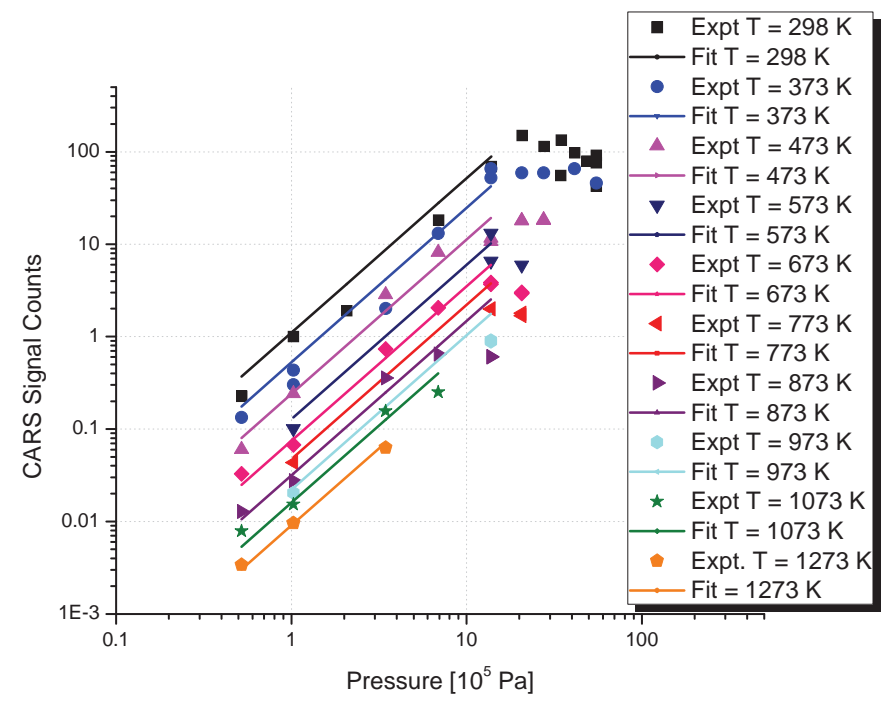

Figure 6. CARS signal intensity versus pressure, for different gas temperatures, measured with a thermocouple. Straight lines are theoretical curves based on Equation 3. 
Figure 7 shows the same CARS signal intensity data graphed on a log-log axis as a function of temperature, with colors indicating different pressures. (The colors in Figures 6 and 7 are unrelated.) As with Figure 6, the data appear linear on this log-log graph, indicating a power law relationship. Consistent with Figure 6, the CARS signal is observed to continuously decrease with temperature. Given the apparent similarity between experimental and theoretical pressure scalings, a least-squares power law fit was implemented in Excel to fit the data. The squared residuals were weighted (divided by) by the square of the value of the fit for each data point to prevent larger signals from dominating the fit. The resulting scaling law based on data in the range of: $0.5<P<14 \times 10^{5} \mathrm{~Pa}$ and $298<T<$ $1400 \mathrm{~K}$ was:

$$
\text { CARS Intensity Relative to STP }=1.7 \times 10^{8} P^{1.67} T^{3.31} \quad \text { (for } P \text { in Pascals, } T \text { in Kelvin) }
$$

The standard error in the fit was $\sim 36 \%$ of the measured values at each fitted point. This law compares well with the computed scalings in Equation 4 and 5 and supports their validity, at least in the range of the measurement. The uncertainty in the exponents, estimated to be $<10 \%$, was determined by repeatedly fitting the data after removing various groups of data points (for example, by excluding a set of measurements having one temperature). Importantly, the measurements cast uncertainty on the validity of using the theoretical scaling of Equation 5 above $14 \times 10^{5} \mathrm{~Pa}$. However, it is possible that an experimental issue, such as beam steering, could be limiting the signal above that pressure. Further experimentation is needed to determine the pressure scaling above $14 \times 10^{5} \mathrm{~Pa}$.

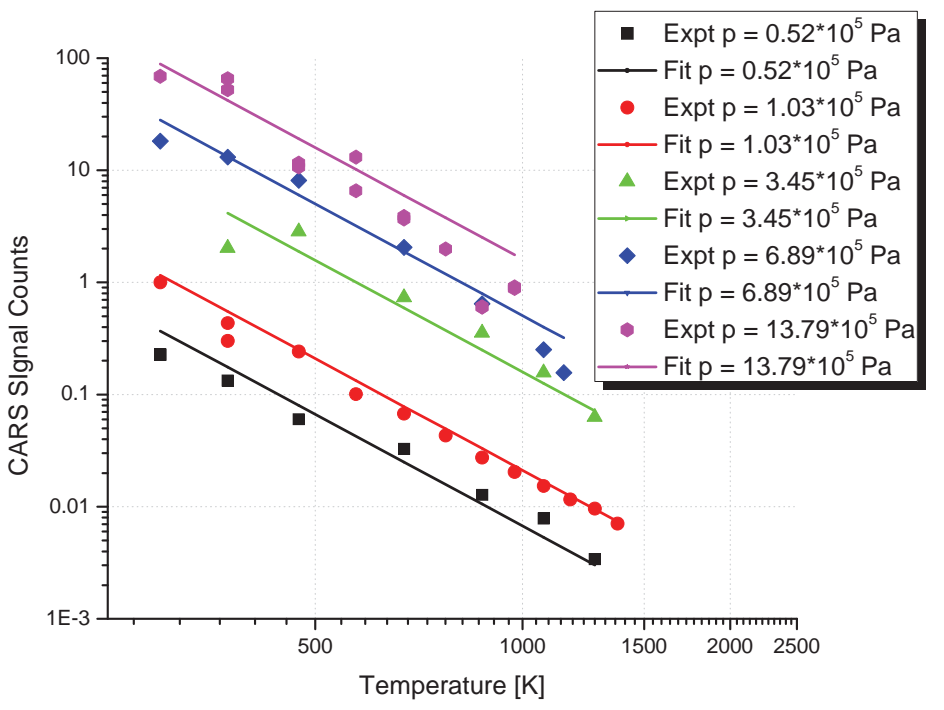

Figure 7. CARS signal intensity versus temperature, for different gas pressures. Only measurements for $P<$ $14 \times 10^{5} \mathrm{~Pa}$ are plotted. Solid lines are theoretical curves based on Equation 6.

Four typical averaged processed $\mathrm{N}_{2}$ spectra are shown in Figure 8; $\mathrm{O}_{2}$ spectra are not shown since the BNNT manufacturing environment is oxygen free and since CARFSFT only has the MEG model implemented for $\mathrm{N}_{2}$. The $\mathrm{N}_{2}$ spectra change shape as environmental conditions vary. As temperature increases, more rotational-vibrational lines appear in the spectrum. As the pressure increases, collisional narrowing occurs, resulting in fewer rotationalvibrational lines being observed. An $\mathrm{N}_{2}$ spectrum at room conditions is provided for comparison. Also shown in these figures is the CARSFT calculation at the nominal measured pressure. For the first three cases, the calculation is at the thermocouple-measured temperature; for the fourth case, the temperature was adjusted for best fit. 

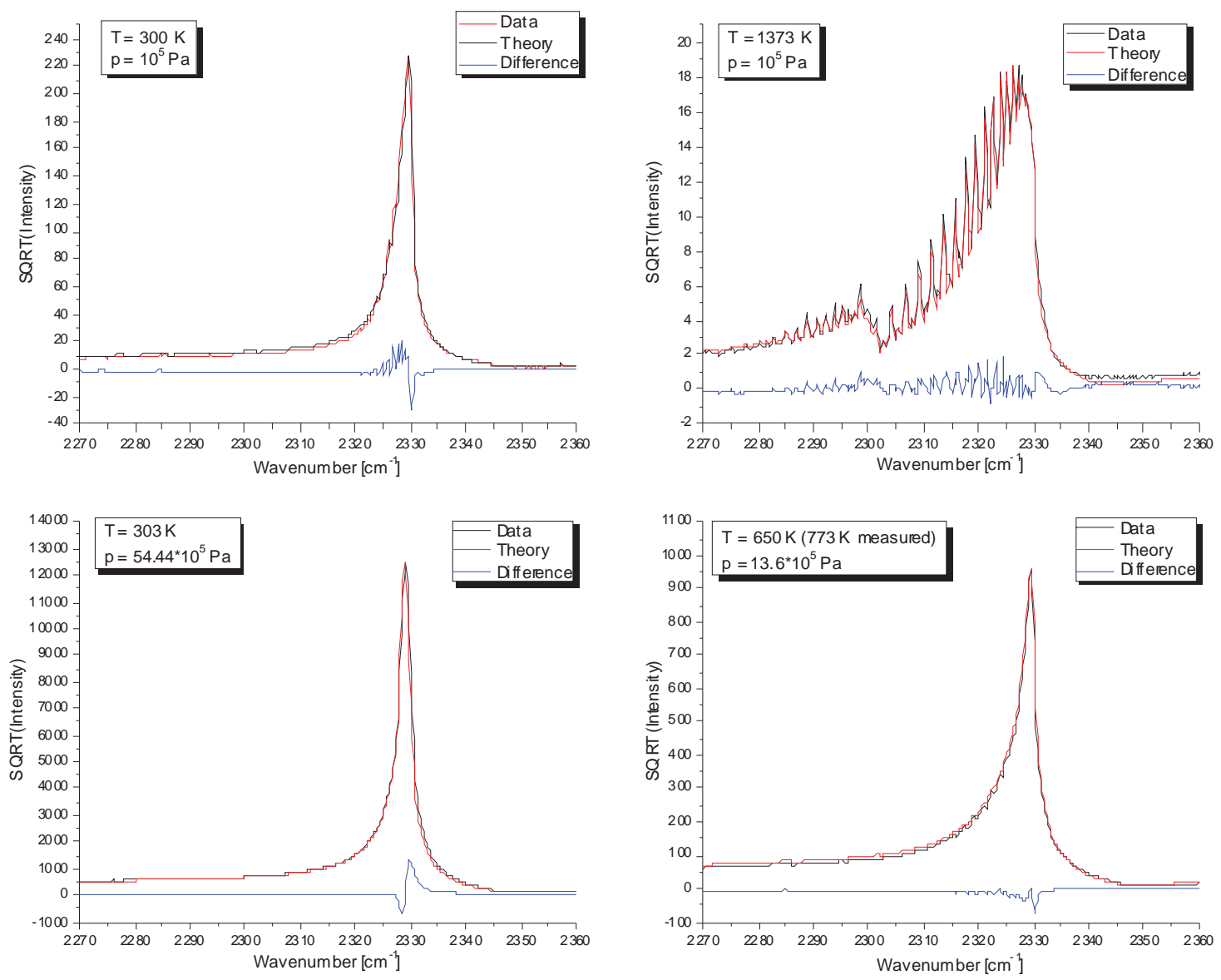

Figure 8. Experimental and fitted CARS spectra. Top left plot: $\mathrm{N}_{2}$ spectrum at room temperature and pressure as a reference spectrum for comparison. Top right plot: $\mathrm{N}_{2}$ spectrum at room pressure and high temperature. Bottom left plot: $\mathrm{N}_{2}$ spectrum at room temperature and high pressure. Bottom right plot: $\mathrm{N}_{2}$ spectrum at high pressure and high temperature.

The first three cases show a good agreement between the calculated and theoretical spectrum. For the fourth case, the calculated spectrum agrees with the measured one at the fitted temperature, but not at the thermocouplemeasured temperature. This last case had pressure and temperature that were both above ambient conditions. Figure 10 shows that such errors in measured temperature occurred whenever both temperature and pressure were raised above ambient. Conversely, accurate temperatures were measured for all temperatures at $P<1$ atm $\left(<1.01 \times 10^{5} \mathrm{~Pa}\right)$ and for all pressures at room temperature. It is speculated that there was stratification in the heater (hotter at top, colder at bottom) when both temperature and pressure were high, causing the observed downward refraction of the laser and signal beams. Thus, the measurement volume may have been substantially below where the thermocouple made its measurement. On the other hand, it is possible that there is an error in the MEG model or its implementation in CARSFT. Further experiments—-perhaps in a more uniformly heated vessel—and further analysis may be required to resolve these discrepancies. 


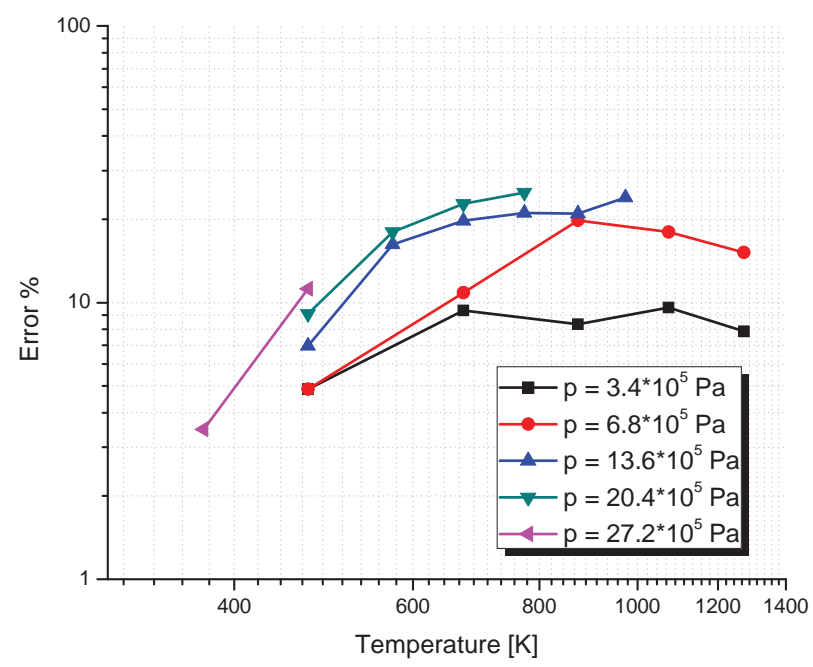

Figure 10. Percentage error between CARS measured temperatures and CARSFT fitted temperatures, at various temperatures and pressures, for selected pressures.

\section{Conclusions}

Dual pump CARS measurements were made in a pressure vessel containing an electrical heater, at pressures up to $55 \times 10^{5} \mathrm{~Pa}$ and temperatures to $1300 \mathrm{~K}$, in the presence of density gradients. An external secondary beam crossing and focal plane imaging system enabled the setup and alignment of the measurement volume inside the pressure vessel. Techniques were demonstrated for rapidly aligning and realigning the signal to the spectrometer in the presence of variable large deflections of the beams path due to density gradients in the heater.

Peak signal intensity was extracted from each spectrum and these intensities were fitted with power-law curves as a function of temperature and pressure, providing experimental support for a previously invalidated theoretical model, at least up to $14 \times 10^{5} \mathrm{~Pa}$. Agreement between the CARS theory using the MEG line shape model and our experimental data was found for the range of pressures and temperatures that the system could achieve, although significant temperature error was observed at conditions where pressure and temperature exceeded ambient simultaneously. Additional work is required to understand and resolve these discrepancies.

The temperature range explored is less than that expected to be encountered in BNNT fabrication (from 290 to $4600 \mathrm{~K}$ ), but the pressure range explored includes the full range expected (from $13 \times 10^{5}$ to $55 \times 10^{5} \mathrm{~Pa}$ ), and equations provided can be extrapolated to allow the signal level at other conditions to be estimated. Other problems that may be encountered in the BNNT environment include laser induced breakdown caused by dust (nanotube) particles in the measurement volume. Our experience working with the heater inside of our pressure vessel is that one initial laser-induced breakdown event can lead to a cascading process of multiple breakdown events, perhaps as shockwaves caused by breakdown then dislodged other particles from the heater wall. This kind of problem might be mitigated by establishing a small continuous purge flow.

\section{References}

${ }^{1}$ P.D. Maker and R. W. Terhune, "Study of Optical Effects Due to an Induced Polarization Third Order in the Electric Field Strength", Applied Physics Lett. 23, (5) pp. 240-242 1973

${ }^{2}$ A. C. Eckbreth, "Laser Diagnostics for Combustion Temperature and Species $2^{\text {nd }}$ Ed", Combustion Science \& Technology Book Series, Vol. 3, Taylor \& Francis, New York, 2002

${ }^{3}$ R. P. Lucht, "Three-laser Coherent Anti-Stokes Raman Scattering Measurements of Two Species", Optic Letters, Vol. 12, No. 2, Feb. 1987, pp. 78-80

${ }^{4}$ M. W. Smith, K. C. Jordan, C. Park, J. Kim,P. T. Lillehei1, R. Crooks and J. S. Harrison, "Very Long Singleand Few-Walled Boron Nitride Nanotubes Via the Pressurized Vapor/Condenser Method", Nanotechnology, Vol. 20, No. 50, 2009 
${ }^{5}$ P.A. Gnoffo, C.C. Fay, "Laser Vaporization and Plume Chemistry in a Boron Nitride Nanotube Production Rig," Journal of Thermophysics and Heat Transfer (2013): 1-13.

${ }^{6}$ K. M. Bultitude, P. M. Danehy, E. Fraval, J. S. Fox, A. F. P. Houwing, "Broadband Coherent Anti-Stokes Raman Spectroscopy (BB_CARS) in Flames and Hypersonic Flows", 2 ${ }^{\text {nd }}$ Australian Conference on Laser Diagnostics in Fluid Mechanics and Combustion, Monash University, Melbourne Australia, 1999

${ }^{7}$ T. Dreier, G. Schiff and A. A. Suvernev, "Collisional Effects in Q Branch Coherent Anti-Stokes Raman Spectra of $\mathrm{N}_{2}$ and $\mathrm{O}_{2}$ at High Pressure and High Temperature", J. Chem. Phys. 100, 1994

${ }^{8}$ T. Seeger, F. Beyrau, A. Bräuer, A. Leipertz, "High pressure pure rotational CARS: comparison of temperature measurements with O2, N2 and synthetic air", Journal of Raman Spectroscopy, Vol. 34, 2003, pp. 932939,

${ }^{9}$ A. D. Cutler, G. Magnotti, L. Cantu, E. Gallo, P. M. Danehy, R. D. Rockwell, C. P. Goyne, J. C. McDaniel, "Dual-Pump CARS Measurements in the University of Virginia's Dual-Mode Scramjet: Configuration "A"”, AIAA-2012-0114, 2012

${ }^{10}$ A. D. Cutler, G. Magnotti, L. Cantu, E. Gallo, P. M. Danehy, R. D. Rockwell, C. P. Goyne, J. C. McDaniel,

"Dual-Pump CARS Measurements in the University of Virginia's Dual-Mode Scramjet: Configuration "C"”, AIAA 2013-0335, 2013

${ }^{11}$ G. Magnotti, A. D. Cutler, P. M. Danehy, "Beam Shaping for CARS Measurements in Turbulent Environments," Applied Optics, Vol. 51, Issue 20, 2012, pp. 4730-4741

${ }^{12}$ A. D. Cutler, G. Magnotti, L. Cantu, E. Gallo, P. M. Danehy, R. D. Rockwell, C. P. Goyne, J. C. McDaniel,

"Dual-Pump CARS Measurements in the University of Virginia's Dual-Mode Scramjet: Configuration "A"”, AIAA-2012-0114, 2012

${ }^{13}$ A. D. Cutler, G. Magnotti, L. Cantu, E. Gallo, P. M. Danehy, R. D. Rockwell, C. P. Goyne, J. C. McDaniel,

"Dual-Pump CARS Measurements in the University of Virginia's Dual-Mode Scramjet: Configuration "C"', AIAA 2013-0335, 2013

${ }^{14}$ R. E. Palmer, "The CARSFT Computer Code for Calculating Coherent Anti-Stokes Raman Spectra: User and Programmer Information", Sandia report SAND89-8206, Feb 1989.

${ }^{15}$ M. Woyde, W. Stricker, "The Application of CARS for Temperature Measurements in High Pressure Combustion Systems", Applied Physics B, Vol. 50, 1990, pp. 519-525

${ }^{16}$ W. D. Kulatilaka, N. Chai, S. V. Naik, S. Roy, N. M. Laurendau, R. Lucht, J. P. Kuehner and J. R. Gord, "Effects of pressure variation on electronic-resonance-enhanced coherent anti-Stokes Raman scattering of nitric oxide", Optic Communications, Vol. 274, 2007 